\title{
Intervención coronaria precoz vs. diferida en pacientes con sindrome coronario agudo sin elevación del segmento ST
}

Early versus delayed invasive intervention in patients with acute coronary syndrome without elevation of ST segment

Metha S y col. N Engl J Med 2009; 360:2165-2175

\section{Objetivo}

Determinar si una intervención coronaria precoz (ICP) -cinecoronariografía (CCG) precoz seguida de una intervención coronaria- es superior a una estrategia diferida (ED) de diagnóstico e intervención en pacientes con síndrome coronario agudo sin elevación del segmento ST (SCASEST).

\section{Diseño, lugar y pacientes}

Ensayo clínico aleatorizado llevado a cabo en 17 países. Participaron 1979 hombres y 1052 mujeres internados por SCASEST dentro de las 24 horas de iniciados los síntomas, candidatos a revascularización coronaria y con dos de los siguientes criterios de alto riesgo: edad mayor a 60 años, cambios isquémicos en el electrocardiograma (infraST de $1 \mathrm{~mm}$, supraST transitorio de $1 \mathrm{~mm}$ ó inversión de ondas T de $3 \mathrm{~mm}$ ) ó elevación de marcadores cardíacos.

\section{Intervención y medición de los resultados principales}

La población fue aleatorizada a ICP (CCG durante las primeras 24 horas, $n=1593$ ) ó ED (CCG luego de 36 horas, $n=1438$ ). Los pacientes fueron revascularizados con angioplastia o cirugía de revascularización miocárdica, según el criterio de equipo tratante. El punto final primario combinado fue la primera ocurrencia de muerte, nuevo infarto o accidente cerebrovascular $(\mathrm{ACV})$ a los seis meses.

\section{Resultados principales}

Se realizó CCG en más del 95\% de los casos (mediana de 14 horas en el grupo precoz y 50 en el diferido). La incidencia a seis meses del punto final primario fue $9,6 \%$ en el grupo de ICP vs. $11,3 \%$ en la rama de ED (HR: 0,85; IC95\% 0,68 a 1,06). Tampoco se registraron diferencias en las tasas de muerte $(4,8 \%$ vs. $5,9 \%$; HR: 0,81 , IC $95 \% 0,60$ a 1,11$)$ infarto $(4,8 \%$ vs $5,7 \%$, $p=0,25)$ y $\operatorname{ACV}(1,3 \%$ vs $1,4 \%, p=0,74)$.

Sin embargo, en los pacientes de más alto riesgo -tercilo superior del escore GRACE1)- la ICP redujo un 35\% la ocurrencia del punto final primario $(13,9 \%$ vs $21,0 \% ; p=0,006)$ sin diferencias entre ambas estrategias en los de los tercilos de menor riesgo.

\section{Conclusiones}

En pacientes con SCASEST, la ICP no se diferenció de la ED en la reducción del punto final primario combinado de muerte, infarto o ACV a los seis meses, salvo en el subgrupo de alto riesgo en quienes la ICP fue superior a la ED.

Palabras claves: síndrome coronario agudo, coronariografía, intervención coronaria. Keywords: acute coronary syndrome, coronariography, coronary intervention. Fuentes de financiamiento: Canadian Institutes of Health Research, Heart and Fuentes de financiamiento: Canadian Institutes of Health Research, Heart
Stroke Foundation of Ontario, GlaxoSmithKline, Sanofi-Aventis y Organon.

\section{Comentario}

A pesar de los avances terapéuticos, los pacientes con SCASEST continúan teniendo una elevada morbimortalidad.

Durante los años noventa, la discusión sobre el manejo de estos pacientes se centró en si debía adoptarse una "estrategia invasiva", con realización rutinaria de una CCG, o una "estrategia conservadora" en la que la CCG se reservaba para quienes evidenciaran isquemia refractaria o inducida en un test de apremio.

Si bien los resultados iniciales de diversos ensayos clínicos fueron algo contradictorios, dada la diversidad de las poblaciones incluidas, la tasa de eventos asociada a los procedimientos y las definiciones de infarto empleadas, los últimos meta-análisis ${ }^{2-3}$ coincidieron en demostrar que en los enfermos de alto riesgo la estrategia invasiva reduce los eventos cardiovasculares, lo que sustenta las recomendaciones de las guías actuales ${ }^{4}$. Desde ese entonces el debate se desplazó desde "¿cateterizar o no cateterizar?" hacia "¿cuándo cateterizar?". En este sentido, la información proveniente de estudios de pequeño tamaño y de diferentes registros que habían intentado contestar esta pregunta todavía no era concluyente ${ }^{5-7}$.

\section{Conclusiones del comentador}

El estudio que hemos resumido fue el primero en analizar de manera multicéntrica cuál es momento óptimo para la intervención coronaria. Sus resultados ponen de manifiesto la importancia de estratificar adecuadamente el riesgo de los pacientes con SCASEST, ya que en aquellos de muy alto riesgo, la intervención precoz es beneficiosa y segura, lo que respalda que sean intervenidos dentro de las 24 horas. Por el contrario, en los de menor riesgo (dos tercios de los pacientes con SCASEST) diferir el estudio angiográfico con eventual revascularización más allá de las 36 horas representa una alternativa de probada eficacia, válida, segura y particularmente aplicable en centros que no disponen de hemodinamia de urgencia.

Ezequiel Berlante [ Senvicio de Cardiología del Hospital Italiano. ezequiel.berlante@hospitalitaliano.org.ar ]

Berlante E. Intervención coronaria precoz vs. diferida en pacientes con síndrome coronario agudo sin elevación del segmento ST. Evid Act Pract Ambul. 12(4). 126. Oct-Dic. 2009. Comentado de: Metha S y col. Early versus Delayed Invasive Intervention in Acute Coronary Syndromes. N Engl J Med 2009; 360:2165-2175. Disponible en URL: http://content.nejm.org/cgi/reprint/360/21/2165.pdf (último acceso 21/07/09).

Referencias

1.Fox K y col. Prediction of risk of death and myocardial infarction in the six months following presentation with acute coronary syndrome: a prospective, multinational, observational study (GRACE). BMJ 2006;333:1091.

2. Mehta S y col. Routine vs selective invasive strategies in patients with acute coronary syndromes. A collaborative meta-analysis of randomized trials. JAMA. 2005;293:2908-2917. 3. O'Donoghue M y col. Early invasive vs conservative treatment strategies in women and men with Unstable Angina and non-ST-segment elevation myocardial infarction A Meta-analysis. JAMA. 2008;300(1):71-80

4. Anderson J y col. ACC/AHA 2007 guidelines for the management of patients with unstable angina/non ST-elevation myocardial infarction: a report of the American College 4. Anderson J y col. ACC/AHA 2007 guidelines for the management of patients with unstable angina/non ST-elevation myocardial infarction: a report of the American College
of Cardiology/American Heart Association Task Force on Practice Guidelines (Writing Committee to Revise the 2002 Guidelines for the Management of Patients With Unstable Angina/Non ST-Elevation Myocardial Infarction). Circulation 2007;116(7):e148-e304.

Downloaded from www.nejm.org on May 27, 2009. Copyright @ 2009 Massachusetts Medical Society. All rights reserved

5 . Neumann F y col. Evaluation of prolonged antithrombotic pretreatment ("coolingoff"strategy) before intervention in patients with unstable coronary syndromes: a randomized controlled trial. JAMA 2003;290:1593-9.

6. Ryan J y col. Optimal timing of intervention in non-STsegment elevation acute coronary syndromes: insights from the CRUSADE (Can Rapid risk stratification of Unstable angina patients Suppress ADverse outcomes with Early implementation of the ACC/AHA guidelines) Registry. Circulation 2005;112:3049-57.

7 .Swanson N y col. Delay to angiography and outcomes following presentation with high-risk, non-ST-elevation acute coronary syndromes: results from the Global Registry of Acute Coronary Events. Heart 2009; 95:211-5. 